\title{
Les enjeux actuels des études de population : une analyse des thèses de doctorat soutenues en France
}

\author{
MYRIAM DE LOENZIEN ${ }^{1}$ \\ GÉRALDINE DUTHÉ2 \\ LAMA KABBANJI ${ }^{1,2}$ \\ ARMELLE ANDRO 3,2 \\ DOMINIQUE DIGUET ${ }^{2}$ \\ CATHERINE SLUSE ${ }^{2}$ \\ DELPHINE ARNOUX ${ }^{4}$
}

\begin{abstract}
Résumé
1. IRD-CEPED.
2. INED.
3. Université Paris I Panthéon Sorbonne.
4. CNF, UIESP.

Cet article vise à explorer le champ des études de population en France à travers le contenu des thèses de doctorat soutenues dans ce pays au cours de la dernière décennie (2000-2012). Nous utilisons pour cela une base de données regroupant 943 thèses. Les analyses menées sont issues de modèles multivariés et de méthodes de statistique textuelle. Les résultats montrent la présence majoritaire de thèses réalisées en référence principalement à la sociologie et dans une moindre mesure à la démographie. La migration constitue la thématique la plus fréquemment traitée. Elle est principalement abordée en lien avec la culture, l'identité, la religion, les minorités, de façon qualitative, ou avec les notions d'environnement, de territoire, de logement. Les études sur la mortalité et la santé ou sur la dynamique de population adoptent généralement une démarche plus quantitative et de modélisation tandis que celles portant sur la fécondité, la famille, la sexualité et la nuptialité occupent une position intermédiaire, proche des études de genre. La scolarisation et l'enseignement sont quant à eux traités en lien avec différentes thématiques, sans prépondérance de l'une d'entre elles. Cette première exploration de la 
base des thèses apporte ainsi des éléments déterminants pour mieux comprendre le contour des études de population ainsi que la manière dont les disciplines se complètent en leur sein. À plus long terme, elle devrait être étendue à une période plus longue et pourrait servir au développement d'une base de données internationale sur les thèses de doctorat en études de population.

\title{
Mots-clés
}

Démographie, thèses de doctorat, étude de population, analyse textuelle, base de données bibliographique, sciences sociales

\begin{abstract}
This article aims at exploring the field of population studies in France through the lens of doctoral theses defended in this country during the last decade (20002012). We rely on the analysis of a database of 943 doctoral theses. Analyses performed include multivariate modeling and textual analysis. Results show that the theses refer mainly to sociology and to a lower extent to demography. Migration is the most frequently researched topic. It is mainly associated either with culture, identity, religion, minorities using qualitative approaches or oriented towards the notions of environment, housing and territory. In contrast, studies on mortality and health or population dynamics tend to involve more quantitative approach and modeling whereas fertility, family, nuptiality and sexuality are often analyzed in conjunction with gender relations, in an intermediate position. Teaching and schooling are equally linked with many topics. This first exploration of the theses database provides insights into population studies boundaries as well as the way disciplines complement each other in this field. In the long term, it could be extended to a longer period and set the basis for the development of a comparative international database of doctoral theses in population studies.
\end{abstract}

\section{Keywords}

Demography, doctoral theses, population study, textual analysis, bibliographic database, social sciences

\section{Approcher la démographie par les thèses en étude de la population}

D'un point de vue historique, les études de population et en particulier la démographie, occupent une place particulière et relativement importante dans le débat social et politique en France (Rosental, 2003). Cependant, si la discipline démographie est bien définie en tant que telle étude des comportements démographiques, de leurs causes et de leurs conséquences -, son champ de recherche est difficile à limiter parce qu'il est très perméable aux autres sciences sociales, aux mathématiques, ou aux sciences de la nature. Pour recouvrir l'ensemble de ses champs et des disciplines concernées, on parle d'études de population (Population 
Studies). Les membres de l'Union internationale pour l'étude scientifique de la population (UIESP) qui est l'association internationale fédérant les scientifiques (étudiants, chercheurs, universitaires, experts) menant des études dans le domaine de la population ont ainsi été consultés en 2000 et 2009 sur leur vision de la démographie, montrant une grande diversité des formations, des approches, des thématiques, etc. (Loriaux, Vichnevskaia, 2006 ; van Dalen, Henkens, 2012). Au niveau académique, le travail doctoral s'appuie sur des savoirs et expériences passées tout en innovant à travers des problématiques et/ou démarches inédites. Il contribue à structurer la recherche et constitue un angle privilégié d'approche de l'état d'une discipline et de son évolution. Le Comité national français (CNF) de l'UIESP a mené en 2013-2014 une réflexion sur les contours des études de population dans le domaine académique et en particulier doctoral en ce début de siècle : quelles sont les thématiques dominantes ? Quels sont les principaux questionnements? En termes de disciplines, de sujets traités, comment se situent au sein des études de population, les travaux qui relèvent plus spécifiquement de la démographie en tant que discipline?

Il existe peu de travaux apportant des éléments de réponse. Or depuis quelques années, l'accès généralisé à de vastes bases de données bibliographiques sur les travaux réalisés a ouvert de nouvelles perspectives de recherche. Les outils informatiques permettent en outre une identification individuelle de chaque thèse soutenue et le recueil d'informations qui lui sont spécifiques. Cette double opportunité permet de constituer un corpus de données sur la production académique. Quelques chercheurs ont notamment étudié le développement de la recherche qualitative (Baribeau, 2007) ou la façon dont sont abordées les migrations internationales en France (Benabou-Lucido, 2011).

La réforme universitaire de 1984 a marqué l'unification des diplômes, créant un doctorat unique et mettant fin à la distinction entre doctorat de troisième cycle et doctorat d'État. Dans cette étude, nous nous sommes intéressées aux thèses soutenues dans les universités et écoles doctorales françaises dans le domaine des études de la population depuis 2000. Pour cela, nous avons dû définir le champ de ces thèses en élaborant une méthodologie à partir de mots-clés, explicitée dans la partie suivante. Les résultats présentés dans cet article traitent plus particulièrement des mots utilisés dans les titres des thèses, puis de manière plus analytique des thématiques et des disciplines dont relèvent ces thèses. 


\section{Constitution et analyse d'une base de données bibliographique des thèses}

Le corpus des thèses soutenues en France dans le domaine des études de population a été constitué à partir du catalogue collectif français alimenté par les bibliothèques et centres de documentation de l'enseignement supérieur et de la recherche : le Sudoc (Système universitaire de documentation $)^{5}$. Les thèses sélectionnées sont les références bibliographiques qui mentionnent au moins le terme «doctorat» dans la variable «note de thèse», variable qui indique également le champ disciplinaire de l'école doctorale, l'université et l'année de soutenance.

Pour identifier les thèses dans le domaine des études de la population, nous avons choisi de procéder par une sélection thématique. Le service de la documentation de l'Institut national d'études démographiques (INED) où cette extraction a été menée utilise une version adaptée et mise à jour de la troisième édition du thesaurus POPIN (CICRED, 1993), un thesaurus multilingue de population permettant l'indexation internationale et standardisée des références bibliographiques en démographie. Celui-ci a été adapté à RAMEAU ${ }^{6}$ qui est le langage d'indexation du Sudoc (par exemple, les thèses traitant de «planification familiale» dans POPIN sont classées dans «régulation des naissances» dans RAMEAU). Les thèses sélectionnées sont donc celles dont l'une des variables de la base du Sudoc (le sujet, le titre, le résumé ou les mots-clés indexés) contient au moins un des mots-clés RAMEAU retenus (voir liste en Annexe 1).

Après sélection dans le Sudoc, les références ont été importées dans le logiciel de gestion bibliographique Zotero. Nous avons éliminé les doublons liés aux thèses extraites via différents mots-clés. Après ce dédoublonnage, nous avons procédé à un examen systématique de plus d'un millier de thèses afin de ne retenir que celles portant sur les populations humaines. Ensuite, les études des populations contemporaines devaient comporter un minimum d'approche «en" population à l'échelle individuelle (recourant par exemple à des entretiens, enquêtes, fichiers administratifs) ou agrégée (contenant par exemple des indicateurs, données de stock et de flux, données de cohortes). Cela exclut certaines formes de sources telles que les récits de vie «fictifs» utilisés en littérature et les

5. L'agence bibliographique de l'enseignement supérieur (Abes) publie également les thèses de doctorat sur le site theses.fr extraites du catalogue Sudoc. Disponible depuis fin 2011, cette base de données est en constante évolution.

6. Répertoire d'autorité-matière encyclopédique et alphabétique unifié. 
Myriam de Loenzien, Géraldine Duthé, Lama Kabbanji, Armelle Andro,

Dominique Diguet, Catherine Sluze, Delphine Arnoux

études de cas individuel que l'on trouve entre autres en psychiatrie ou en médecine. Les thèses de démographie historique, où des sources originales sont utilisées, ont été conservées ainsi que des thèses purement méthodologiques proposant des modèles populationnels. Toutes les exclusions opérées à cette étape ont été discutées au sein de l'équipe. Au final, notre analyse porte sur 943 thèses soutenues entre 2000 et 2012.

Une fois le corpus de thèses constitué, les informations disponibles dans le Sudoc ont été exploitées. Comme le langage et les mots employés occupent une place prépondérante, nous avons mené une analyse textuelle des titres des thèses qui résument les sujets abordés et les approches adoptées par ces travaux, dans l'esprit de ce qui a pu être fait par ailleurs sur d'autres corpus bibliographiques (Guérin-Pace, Saint-Julien, 2012). Cette analyse permet d'étudier la fréquence des termes utilisés, après lemmatisation, et la constitution d'un graphe de similitude en recourant au module Iramuteq du logiciel R (Marchand et al., 2012). Pour faciliter la lecture du graphe, les mots outils ont été éliminés (articles, adjectifs non qualificatifs, mots de liaison...). Nous n'avons représenté que les arêtes pour lesquelles la valeur de l'indice de similitude est inférieure ou égale 3.

Le titre, le résumé, les mots-clés indexés ont permis de définir les grandes thématiques abordées. Celles-ci ont été regroupées en onze modalités pour cette analyse. Par exemple, les thèses sur la migration incluent celles portant sur les migrants, sur les causes, conséquences et les conditions de la migration, sur le devenir des migrants de deuxième génération ou encore des étrangers. Ce classement n'est pas exclusif, une même thèse pouvant relever de plusieurs thématiques. Nous avons ensuite mené une analyse en composantes principales afin de situer les thèses les unes par rapport aux autres en tenant compte des thématiques abordées dans chacune d'elles. Cela permet en outre de dégager des convergences et divergences entre thématiques.

Dans le Sudoc, une variable «discipline» indique la discipline telle qu'elle figure sur la page de titre (ou son équivalent) de la thèse. Cette indication représente le plus souvent la discipline principale dans laquelle est soutenue la thèse, mais elle peut être plus ou moins précise, et reprendre par exemple l'intitulé de l'école doctorale. Cette variable a été recodée pour homogénéiser les disciplines auxquelles elle fait référence, par exemple «sciences économiques» a été recodée en «économie» (voir la liste en Annexe 2). Seule la modalité «Étude régionale» qui renvoie à des approches en termes d'entité socioculturelle (par exemple études anglophones, études ibériques) ne caractérise pas la ou les approches disci- 
plinaires utilisées dans la thèse. Par ailleurs, cette variable discipline peut prendre plusieurs modalités pour une même thèse.

\section{Les mots les plus fréquemment utilisés dans les titres des thèses}

L'analyse textuelle des titres des thèses se fonde sur 14553 occurrences, 2612 mots lemmatisés ${ }^{7}$ et 1565 hapax $^{8}$. La Figure 1 présente le nuage des 150 mots lemmatisés les plus fréquents dans les titres. Plus cette fréquence est élevée, plus la taille de police est importante et le positionnement du mot central dans le nuage. Cette représentation met en évidence la grande diversité du vocabulaire qui s'exprime par une forte dispersion des mots dans le nuage, la proportion la plus élevée ne dépassant pas $5 \%$. Cette dispersion apparaît aussi dans la fréquence relativement forte d'hapax (60\% des mots lemmatisés, $11 \%$ des occurrences). Neuf mots se distinguent toutefois : «France» $(6,0 \%$ des mots lemmatisés), «migration» $(3,9 \%)$, «immigration» $(3,4 \%)$, et dans une moindre mesure «politique» $(3,2 \%)$, «social» $(3,1 \%)$, «population» $(2,9 \%)$, «étude» $(2,8 \%)$, «cas» $(2,6 \%)$, «analyse» $(2,5 \%)$ et «démographique» $(2,0 \%)$. Les autres mots ont une fréquence qui reste faible (inférieure à $2,0 \%)$.

Malgré une relative diversité, ce résultat permet de dégager plusieurs points : une visibilité des thèses portant sur la France; des sujets portant sur la migration plus nombreux par rapport à d'autres sujets d'étude de population tels que la mortalité et la fécondité ; l'inscription dans des perspectives qui privilégient les dimensions sociodémographiques et/ou politiques; des méthodologies centrées sur des études de cas qui révèlent un intérêt pour des approches empiriques ou encore des études à vocation analytique.

7. Les verbes sont ramenés à l'infinitif, les noms au singulier et les adjectifs au masculin singulier.

8. Les hapax sont des mots qui n'apparaissent qu'une seule fois. 
Figure 1 Nuage des 150 mots lemmatisés les plus fréquents dans les titres des thèses

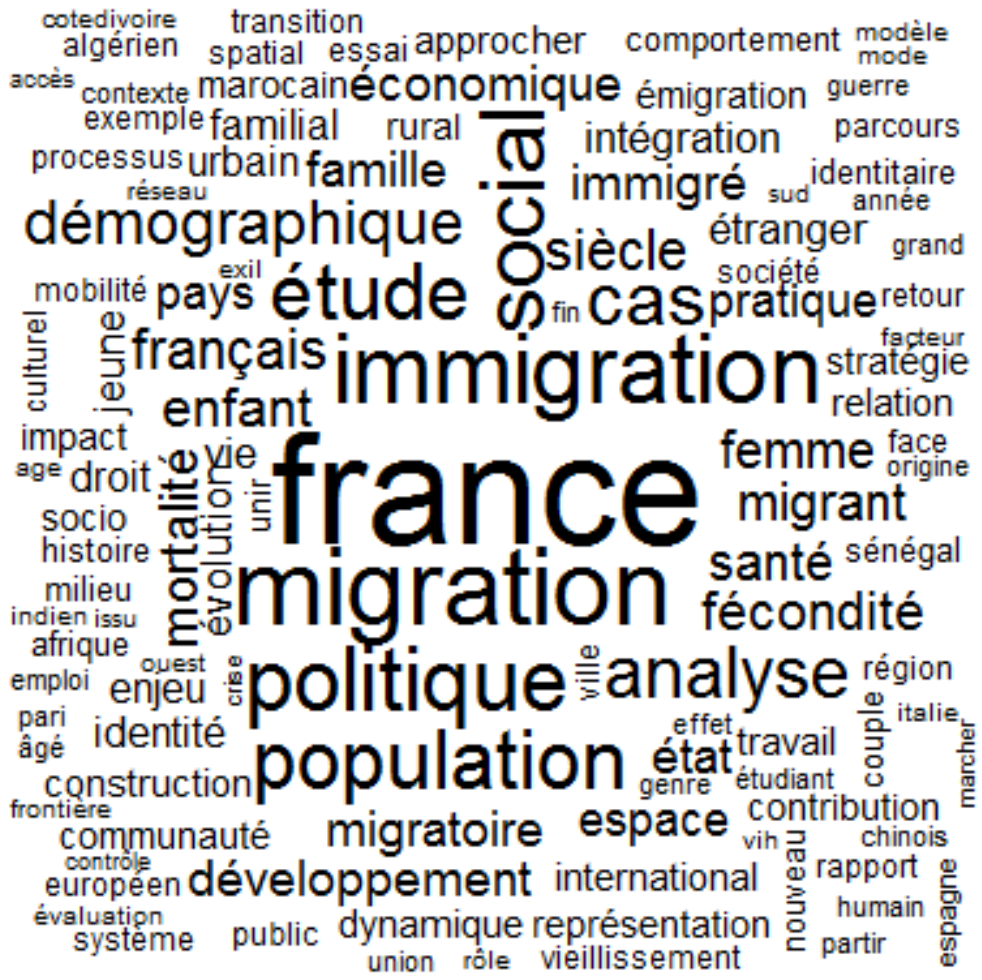

L'arbre des liaisons lexicales (Figure 2) est un graphe de similitude élaboré à partir des cooccurrences des mots lemmatisés dans les titres. Il confirme le fait que les thèses sont fortement centrées sur la France et fait apparaître plusieurs pôles sémantiques secondaires distincts. Deux pôles portent sur la mobilité spatiale et l'identité. L'un est centré autour de la notion de migration (en haut sur le graphique), l'autre autour de celle d'immigration (en bas à gauche sur le graphique). Le premier met en avant la dimension internationale tandis que le deuxième traite de problématiques liées au pays d'accueil. Un deuxième ensemble renvoie à une distinction plutôt disciplinaire. Il est constitué autour des notions de population d'une part (à gauche sur le graphique), d'aspects sociaux d'autre part (en bas sur le graphique). Les deux derniers sont plutôt méthodologiques autour des notions de cas associés à l'Afrique subsaharienne (en haut sur le graphique) et l'analyse (à droite sur le graphique). 
FIgURE 2 Arbre des liaisons lexicales des titres des thèses

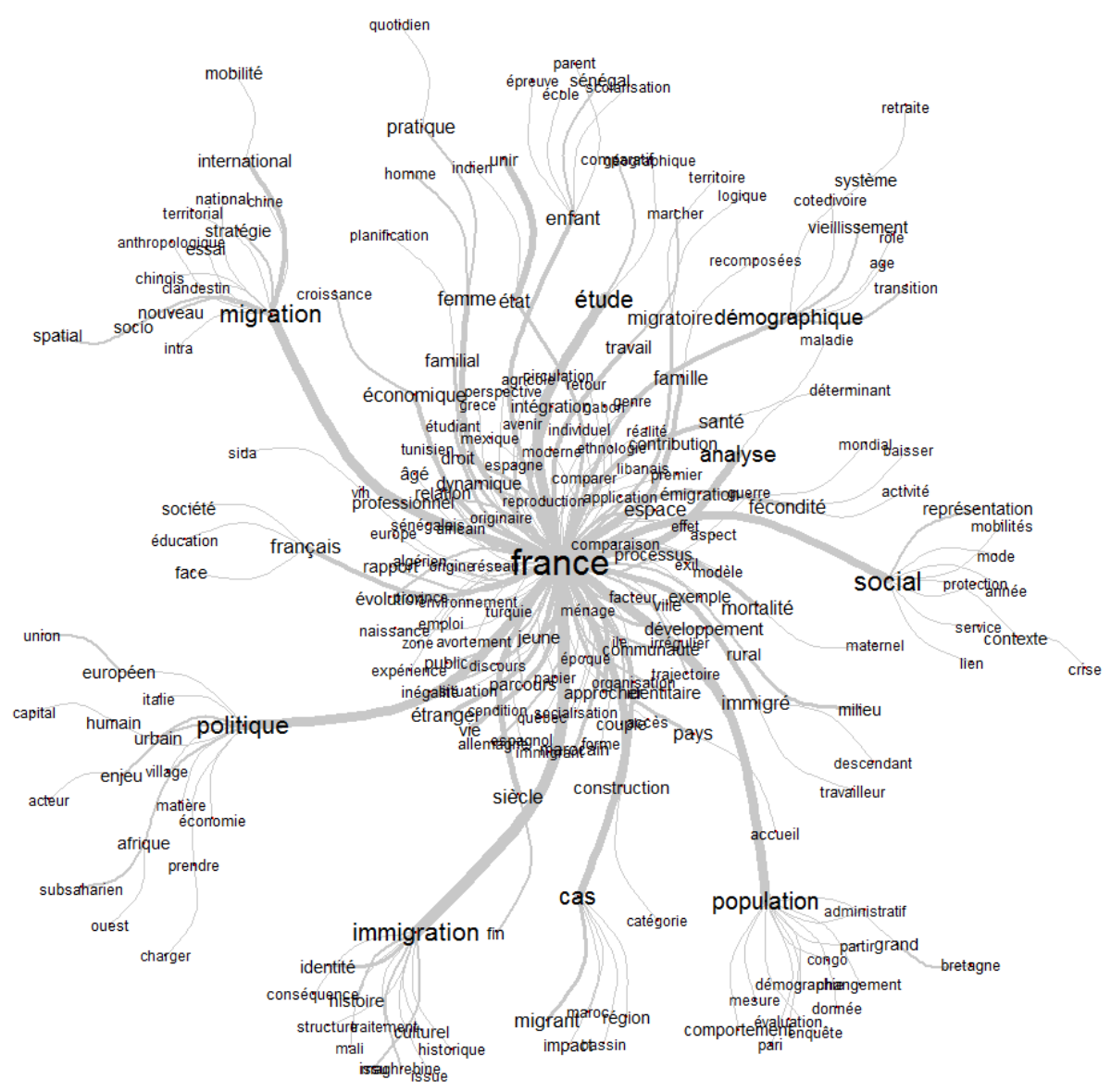

\section{Des pôles thématiques classiques et de multiples positionnements}

Au-delà des mots du titre des thèses, une analyse des grandes thématiques nous permet de mieux approcher leur contenu. Les thèses n'abordant qu'une seule thématique sont minoritaires (16\%). Dans près de la moitié des cas ( $44 \%$ ), les thèses en couvrent deux, et trois dans un tiers des cas $(29 \%)$. Cette distribution met ainsi en avant le caractère plurithématique des thèses. 
De manière cohérente avec l'analyse textuelle menée sur les mots du titre, la thématique la plus représentée est la migration qui est traitée dans plus de la moitié des thèses ( $52 \%$, Figure 3$)$. Elle fait référence à divers types de migrations et d'immigrations (saisonnières, clandestines, de retour, de transit, forcées, de travail, exil, exode, diaspora) à différentes échelles. Une deuxième thématique est constituée de sujets qui ont trait à la culture, aux questions d'identité, de minorités, de socialisation, de religion et d'ordre social. Un peu plus d'un tiers des thèses sont concernées (35\%).

FigURE 3 Part de chaque thématique, catégories non exclusives

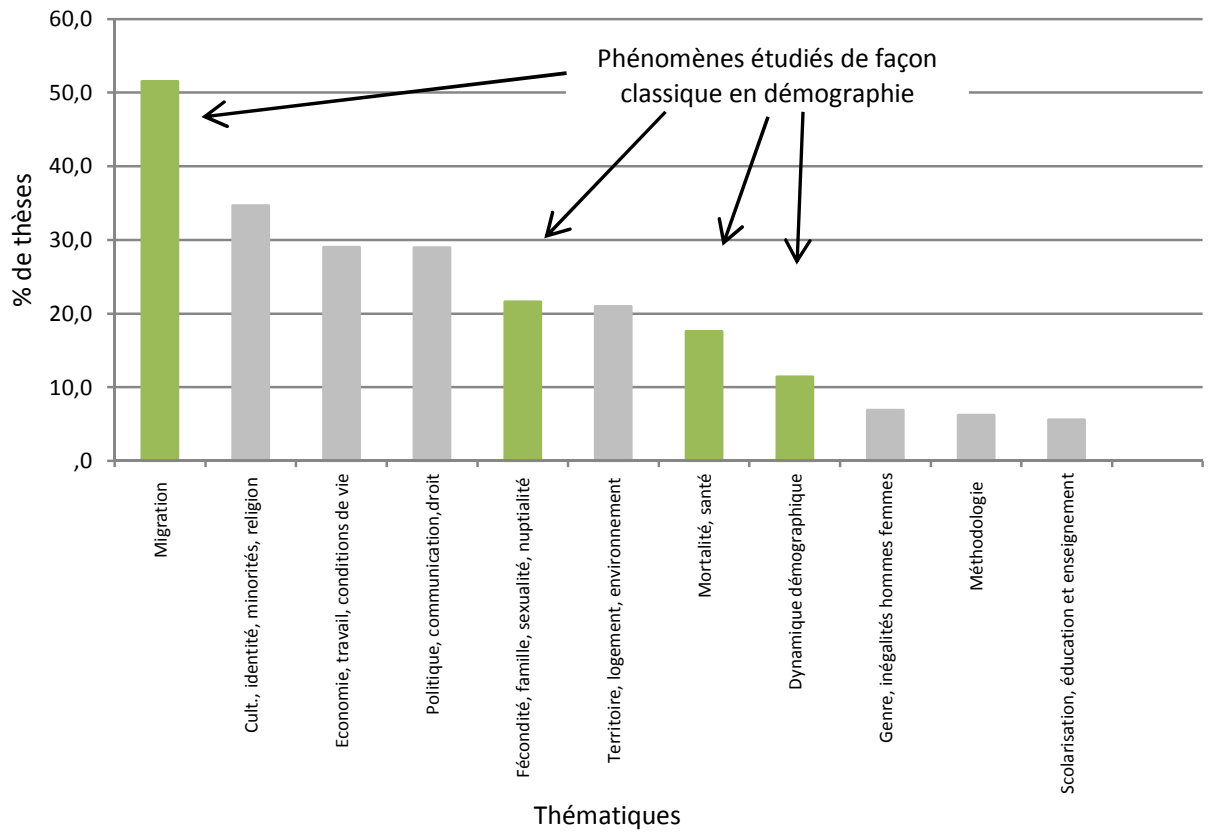

Source : base de données des thèses de doctorat constituée par les auteures.

Deux thématiques regroupent chacune près d'un tiers des thèses. L'une comprend des sujets concernant les approches économiques, le travail et les conditions de vie (29\%). Elle décrit les interactions entre phénomènes démographiques et économiques, les comportements démo-économiques des familles, notamment en matière de consommation ou d'activité. Elle aborde tout ce qui est en rapport avec l'emploi ou les conditions matérielles à des échelles micro- ou macro-économiques. L'autre 
rassemble des questions relatives à la politique, au droit ou à la communication $(29 \%)$.

Plus d'une thèse sur cinq (22 \%) porte sur la fécondité, l'union, les structures familiales ainsi que l'évolution de l'environnement familial dans lequel grandissent les enfants. Ces thèses s'intéressent également aux comportements sexuels et reproductifs, contraceptifs ainsi qu'au désir d'enfants, à la fertilité et l'avortement. La mortalité et la santé, thématiques qui occupent une place centrale en démographie, prennent une place moindre dans ce corpus de thèses $(18 \%)$. Ce groupe inclut les études des tendances de la mortalité et de l'état de santé des populations, la mesure et l'analyse des causes de décès, la santé perçue.

Les autres thématiques correspondent en grande partie à des approches méthodologiques ou des dimensions plus transversales. Il s'agit de l'étude de la dynamique démographique qui décrit les structures et évolutions de la population (12\%), des études de genre (7\%) et des travaux essentiellement méthodologiques $(6 \%)$. Enfin, les questions relatives à la scolarisation, l'éducation et l'enseignement sont plus rares (6\%).

Pour représenter la multiplicité des thématiques abordées dans une même thèse, nous utilisons l'analyse en composantes principales (ACP) (Figure 4). Celle-ci permet en effet de mettre en relation les onze thématiques les unes par rapport aux autres et de situer les thèses par rapport à elles. L'ACP permet de dégager quelques dimensions analytiques. Nous nous intéressons principalement aux deux premiers plans factoriels qui résument $42 \%$ de l'information (Annexe 3 ). Sur le graphique, chaque point représente un ensemble de thèses (la taille du point augmente avec leur effectif, selon un regroupement par classes, voir Annexe 3). Le nuage paraît relativement étalé, ce qui témoigne d'une diversité des combinaisons thématiques.

Le premier plan contient près d'un tiers de l'information (31\%). Il fait apparaître deux grandes oppositions (Annexe 3). La première, matérialisée par l'axe horizontal établit une distinction entre deux types d'études. Sur la gauche du graphique figurent les thèses portant plutôt sur la migration, favorisant des problématiques relatives à l'identité, aux minorités et la religion, dont une part non négligeable s'intéresse notamment aux dimensions politiques, législatives, juridiques et de communication. À l'opposé, sur la droite, les travaux concernent davantage la mortalité, la santé, l'épidémiologie et la dynamique des populations, avec une attention particulière portée sur les approches méthodologiques et la modélisation. Ce premier axe est donc significatif d'un choix de phénomènes sociaux étudiés, mais aussi d'un arbitrage entre approches plus qualitatives à gauche et plus quantitatives à droite. Dans cette dimension, 
les approches de genre et la prise en compte de l'environnement ou du territoire occupent une place non spécifique à l'un ou l'autre des termes de l'opposition. Elles ne sont pas plus présentes dans les études de migration que dans celles de mortalité, santé ou dynamiques de population. Par contre, le deuxième axe montre les spécificités de ces deux groupes de thématique.

FIGURE 4 Représentation des thèses dans le premier plan factoriel de l'analyse en composantes principale sur les 11 thématiques

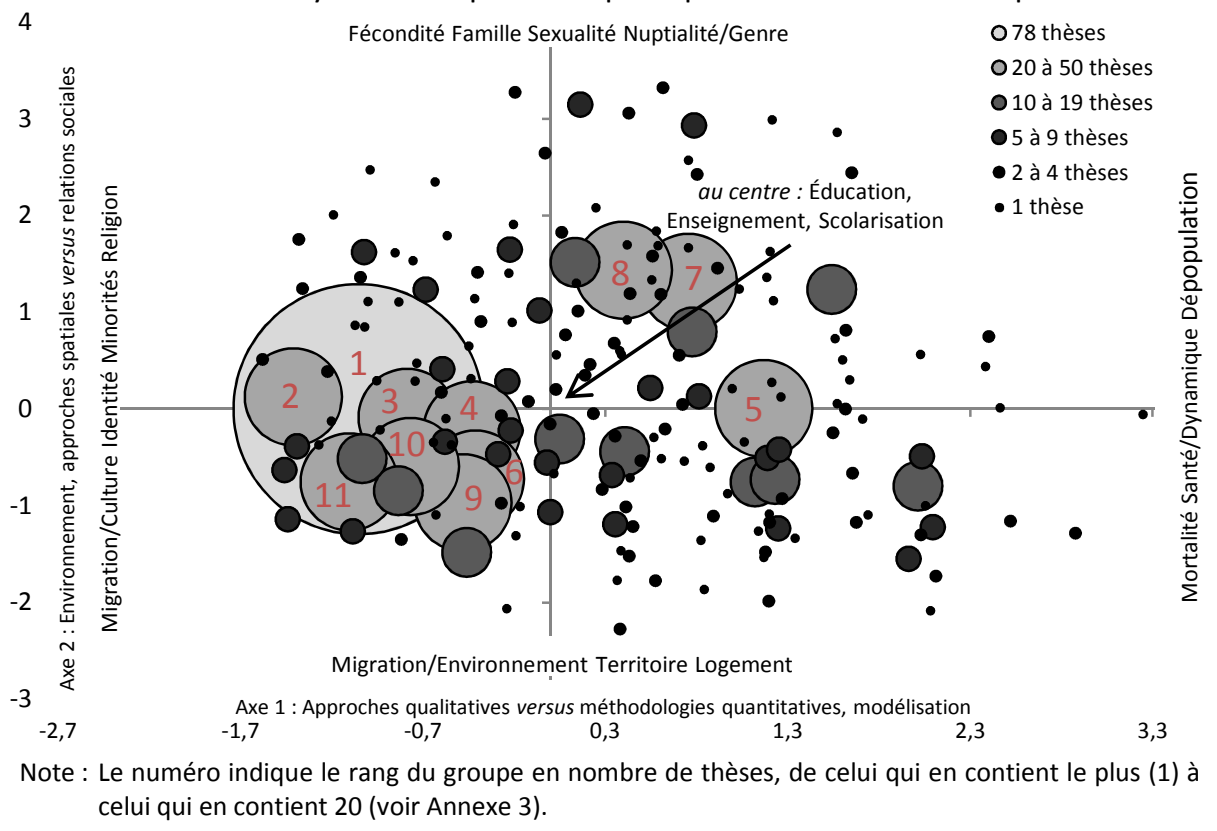

Le deuxième axe, vertical sur le graphique, correspond en effet à une autre distinction. Il privilégie en bas du graphique les études portant sur le territoire, le logement, l'environnement et, dans une moindre mesure, sur le développement économique, le travail et les conditions de vie. Ces thématiques, souvent liées aux problématiques de migration et dans une moindre mesure à des sujets portant sur la santé ou encore la dynamique des populations, sont contrastées avec celles portant sur la fécondité, la famille, la sexualité, la nuptialité situées en haut du graphique (et pour beaucoup, plutôt à droite), de même que celles qui ont trait aux approches de genre et aux inégalités entre hommes et femmes, bien que celles-ci soient moins fortement présentes. 
Seule une thématique occupe une place non spécifique sur ces deux axes: la scolarisation et l'enseignement. Cette spécificité explicite la place centrale des questions relatives à l'éducation pour l'ensemble des études de population.

La prise en compte des axes suivants met en évidence de nouvelles dimensions d'analyse de ce nuage. En particulier, le troisième axe permet de préciser la place de la thématique dont la position est «neutre» pour les deux premiers axes : la scolarisation et l'enseignement. Cette thématique apparaît en effet opposée à des approches en termes de culture, de minorité, d'identité et de religion. Elle est à l'inverse associée à des travaux sur l'économie, le travail et les conditions de vie. Enfin, le quatrième axe oppose clairement les approches politiques, juridiques, les études sur le droit et les législations à celles qui portent sur le territoire, le logement et l'environnement. Les résultats montrent une pertinence limitée de la représentation au-delà du cinquième axe à partir duquel on dispose de plus de la moitié de l'information (61\%).

Cette analyse confirme donc la place prépondérante de la migration, tout en précisant les angles sous lesquels elle est traitée. Elle fait apparaître un ensemble relativement homogène de thèses mettant en avant la culture, l'identité, la religion, les minorités, tandis que l'autre perspective plus orientée vers une analyse en termes d'environnement, de territoire, de logement est marquée par une plus grande diversité thématique. Les études portant sur les autres thématiques sont plus hétérogènes. En particulier, les études sur la mortalité et la santé ou sur la dynamique de population adoptent une démarche plus quantitative et de modélisation. De ce point de vue, les études sur la fécondité, la famille, la sexualité et la nuptialité occupent une position plus nuancée dans une proximité avec l'étude des relations de genre.

\section{Des thèses fortement centrées sur les sciences sociales, mais avec des profils disciplinaires différents selon la thématique}

L'étude des disciplines associées aux thèses permet de préciser les approches adoptées dans les recherches doctorales. Bien que la plupart des thèses $(80 \%)$ ne fassent référence qu'à une discipline, la proportion de celles qui font référence à deux disciplines est relativement élevée (18\%) et une minorité ( $2 \%$ ) en mentionne au moins trois (Annexe 2 ). 
La dernière colonne du Tableau 1 présenté ci-après donne la répartition des disciplines pour l'ensemble des thèses (plusieurs disciplines possibles par thèse). La sociologie est la plus fortement représentée, près d'un tiers des thèses s'y référant (28\%). La démographie n'est explicitement mentionnée que dans $20 \%$ des cas. L'histoire, l'économie ou la géographie caractérisent environ une thèse sur dix. Viennent ensuite les thèses en études régionales (sur une région géographique et/ou une aire culturelle en particulier). Moins de $7 \%$ des thèses relèvent de l'anthropologie, $5 \%$ des sciences politiques et $4 \%$ du droit ou de la santé publique. Près d'une thèse sur dix se réfère à une autre discipline dans des domaines très divers comme les lettres, la biologie, la psychologie, etc. (voir Annexe 2). Ainsi, les thèses se situent pour la plupart dans le champ des sciences sociales.

Parmi les $20 \%$ de thèses pluridisciplinaires, de multiples combinaisons sont possibles. L'analyse relationnelle permet d'en rendre compte visuellement. Sur la Figure 5, chaque lien entre disciplines caractérise l'existence d'au moins une thèse rattachée simultanément à ces deux disciplines. Le graphe prend la forme d'une étoile qui met en évidence les liens qu'entretiennent la sociologie, l'histoire et l'anthropologie avec d'autres disciplines. On y retrouve des affinités relativement classiques, notamment celles se situant en grande partie dans le champ biomédical réunissant la santé publique, la médecine et la biologie, ou encore les liens entre statistique et santé publique. Les liens de la démographie avec les autres disciplines font apparaître de fortes connexions avec la sociologie, l'histoire, la géographie et l'économie. Ses liens avec les autres disciplines sont plus ténus. Enfin, certaines disciplines apparaissent relativement peu connectées, telles que la philosophie et la linguistique qui n'ont de lien qu'avec la sociologie ou encore l'écologie plus marginale puisque ses liens avec les sciences sociales se nouent via la biologie. 
Figure 5 Associations des disciplines au sein des $20 \%$ de thèses pluridisciplinaires (graphe non directionnel de Fruchterman-Reingo)

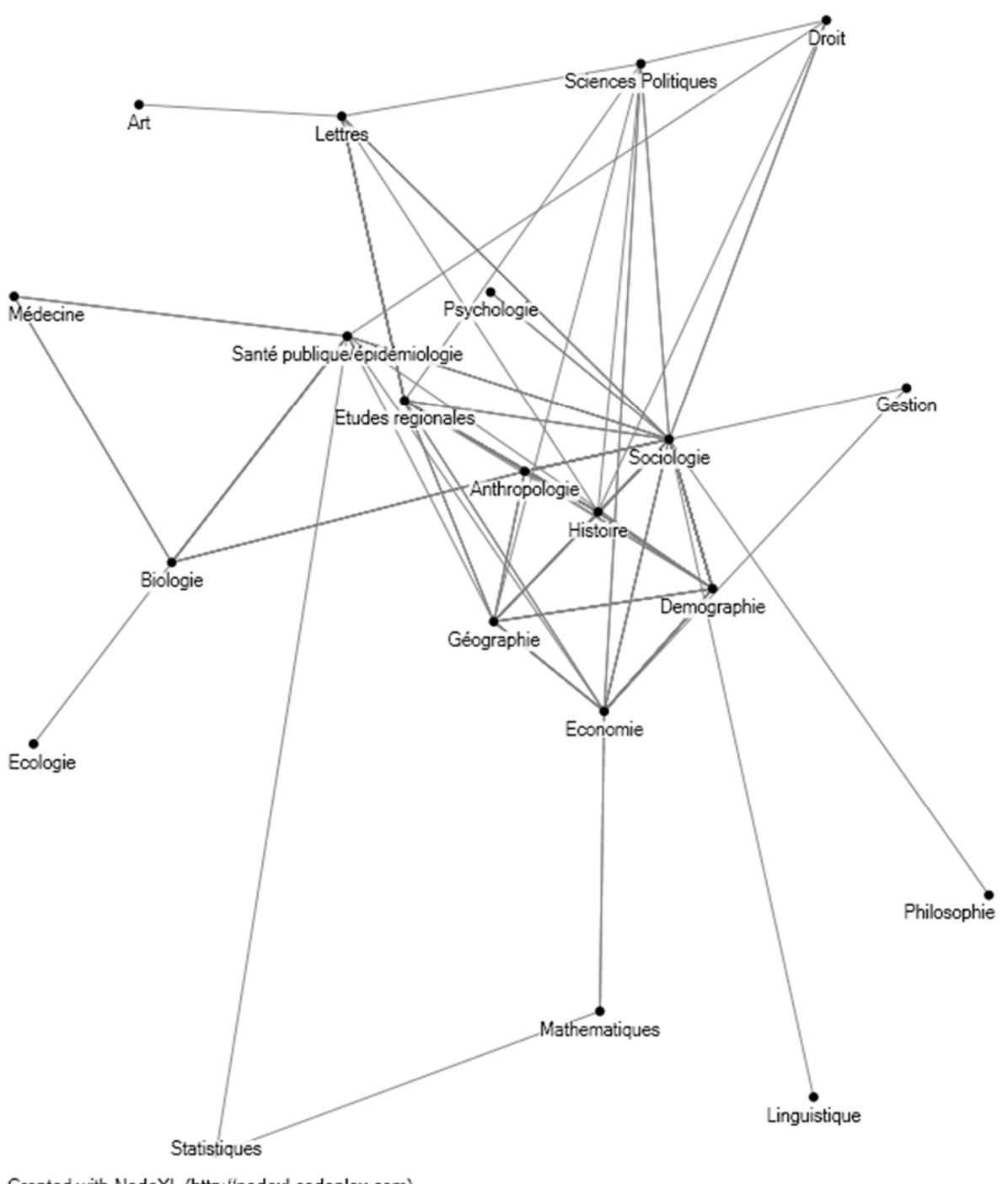

Created with NodeXL (http $/ /$ nodexl.codeplex.com)

Une analyse croisée des disciplines et des thématiques permet de mieux comprendre la représentation de ces différentes disciplines dans les études de population. Pour cela, nous étudions la représentation de chaque discipline dans les thématiques respectives, en l'interprétant de façon relative, c'est-à-dire en la comparant à la place occupée par chaque discipline dans l'ensemble du corpus des thèses. Cette analyse est contenue 
dans le Tableau 1. Celui-ci fait apparaître une distinction entre des thématiques qui entretiennent des liens privilégiés avec certaines disciplines et celles qui se caractérisent par une perspective plus inter ou pluridisciplinaire.

TABleau 1 Pourcentage des thèses mentionnant chaque discipline, selon la thématique traitée (\% par rapport au nombre total de thèses, $n=943$ )

\begin{tabular}{|c|c|c|c|c|c|c|c|c|c|c|c|c|}
\hline Thématique & 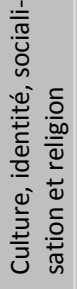 & 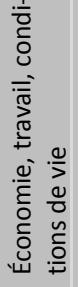 & 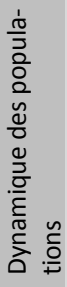 & 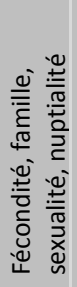 & 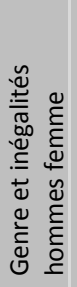 & 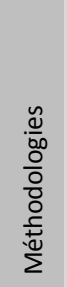 & 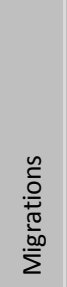 & 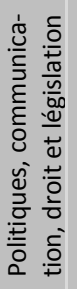 & 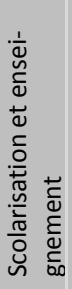 & 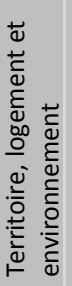 & 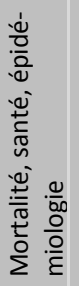 & $\begin{array}{l}\frac{0}{0} \\
\text { है } \\
\Phi \\
\tilde{\omega} \\
\text { ப் }\end{array}$ \\
\hline Sociologie & 39,6 & 21,7 & 10,2 & 34,3 & 46,2 & 15,0 & 33,7 & 23,6 & 26,4 & 21,6 & 15,1 & 28,3 \\
\hline Démographie & 11,9 & 24,3 & 26,9 & 31,4 & 21,5 & 20,0 & 6,2 & 15,3 & 22,6 & 21,6 & 28,3 & 19,5 \\
\hline Histoire & 13,7 & 8,7 & 22,2 & 9,7 & 7,7 & 6,7 & 13,8 & 16,7 & 5,7 & 17,6 & 6,0 & 12,3 \\
\hline Économie & 3,0 & 30,8 & 21,3 & 10,1 & 10,8 & 23,3 & 9,7 & 10,2 & 22,6 & 7,0 & 9,0 & 12,0 \\
\hline Géographie & 11,0 & 13,0 & 7,4 & 3,4 & 10,8 & 1,7 & 12,7 & 7,3 & 5,7 & 33,7 & 8,4 & 10,6 \\
\hline Études régionales & 11,6 & 5,1 & 5,6 & 3,9 & 6,2 & 1,7 & 9,9 & 9,5 & 11,3 & 8,5 & 2,4 & 7,4 \\
\hline Anthropologie & 10,7 & 2,2 & 4,6 & 7,2 & 4,6 & 5,0 & 8,4 & 6,2 & 5,7 & 4,5 & 5,4 & 6,7 \\
\hline Sciences politiques & 4,6 & 4,3 & 1,9 & 1,9 & 0,0 & 1,7 & 9,2 & 13,1 & 5,7 & 2,0 & 2,4 & 5,3 \\
\hline Santé publique & 0,3 & 1,1 & 5,6 & 1,4 & 1,5 & 5,0 & 0,4 & 0,4 & 0,0 & 2,5 & 23,5 & 4,2 \\
\hline Droit & 3,4 & 0,0 & 0,0 & 6,3 & 3,1 & 0,0 & 5,1 & 9,5 & 1,9 & 2,0 & 3,0 & 4,2 \\
\hline Autre & 11,3 & 7,6 & 20,4 & 10,9 & 4,6 & 33,3 & 8,4 & 6,5 & 11,3 & 5,5 & 21,1 & 10,9 \\
\hline
\end{tabular}

Exemple de lecture : $39,6 \%$ des thèses relevant de la thématique «culture...» font référence à la sociologie, $11,9 \%$ des thèses relevant de la thématique "culture...» font référence à la démographie, ces deux disciplines pouvant être présentes simultanément pour une même thèse.

En vert: valeur significativement supérieure à la moyenne; en rouge : valeur significativement inférieure à la moyenne, par rapport au \% pour l'ensemble des thèses (chi-deux, $p<5 \%$ ).

Note : une thèse peut être rattachée à plusieurs disciplines.

Deux thématiques sont fortement marquées par une discipline prédominante. Il s'agit des études de genre qui relèvent, pour près de la moitié des thèses, de la sociologie ( $46 \%$ contre $28 \%$ pour l'ensemble des thèses) et de la thématique regroupant les questions relatives à la culture, l'identité, les minorités et la religion, pour laquelle cette proportion est également élevée ( $40 \%$ des thèses abordant cette thématique font référence à la sociologie).

Plus partagés d'un point de vue disciplinaire, les travaux portant sur la fécondité, la famille, la sexualité et la nuptialité sont marqués par un fort lien avec la sociologie (34\%), mais aussi la démographie (31\% contre 
$20 \%$ pour l'ensemble des thèses). La thématique concernant les aspects économiques est également caractérisée par un rattachement plus large avec $31 \%$ de thèses en économie, $24 \%$ en démographie et $22 \%$ en sociologie (qui est donc sous-représentée par rapport à l'ensemble des thèses). Les thèses traitant de questions de territoire, de logement, et d'environnement relèvent pour un tiers d'entre elles de la géographie (34\%), $22 \%$ de la sociologie et de la démographie, mais aussi de l'histoire, surreprésentée dans cette thématique $(18 \%$ contre $12 \%$ pour l'ensemble des thèses). Les thèses traitant de mortalité, de santé ou d'épidémiologie relèvent pour la plupart de la démographie (28\%) et de la santé publique (regroupée avec l'épidémiologie) (24\%). De même, les études sur les dynamiques de population font appel surtout à la démographie (27\%), à l'histoire (22\%) et à l'économie (21\%). Les études portant sur la scolarisation et l'enseignement sont surreprésentées en économie par rapport à l'ensemble des thèses $(22,6 \%$ contre $12 \%)$, mais leurs liens avec la sociologie et la démographie sont équivalents à celui de l'ensemble des thèses. Ce premier groupe de thématiques n'exclut pas les autres disciplines, mais celles-ci sont moins représentées.

Un troisième groupe est constitué des thématiques caractérisées par une importante diversité disciplinaire. La plus caractéristique est l'étude des migrations : malgré une forte représentation de la sociologie (34\%), sont représentées : l'histoire (14\%), la géographie (13\%), les études régionales $(10 \%)$, l'économie $(10 \%)$, les sciences politiques $(9 \%)$ et l'anthropologie $(8 \%)$. La démographie est particulièrement sous-représentée $(6 \%)$ dans les études sur la migration. De façon similaire, les études portant sur les politiques, la communication et les aspects juridiques sont identifiées comme des thèses en sociologie $(24 \%)$, histoire (17\%), démographie (15\%), sciences politiques (13\%), économie (10\%), droit (10\%) ou études régionales.

Les études à vocation méthodologique sont caractérisées par trois grandes disciplines que sont l'économie $(23,3 \%)$, la démographie $(20 \%)$ et dans une moindre mesure la sociologie $(15 \%)$, mais un tiers relèvent d'autres disciplines non spécifiées dans ce tableau (voir Annexe 2).

\section{Profils thématiques des disciplines}

Une lecture alternative du tableau 1 permet de dresser un profil des disciplines selon les thématiques qu'elles traitent (Tableau 2). Cet autre regard indique que les disciplines représentées dans les études de popula- 
tion sont chacune fortement investies dans quelques thématiques. Les thèses se référant à la sociologie traitent de façon massive de la migration ( $61 \%$ des thèses) et de la thématique qui lui est liée portant sur la culture, l'identité, la socialisation et la religion. De même, l'économie développe des problématiques relatives aux aspects économiques, au travail, aux conditions de vie (75\%) et dans une moindre mesure aux migrations (41,6\%). L'anthropologie et les études régionales présentent des profils similaires, avec un intérêt marqué pour les migrations (respectivement $65,1 \%$ et $68,6 \%$ ) ainsi que la culture, l'identité, la socialisation et la religion (respectivement $55,6 \%$ et $54,3 \%$ ). Le constat est valable aussi pour les autres disciplines. L'ensemble de ces disciplines aborde par ailleurs d'autres thématiques qui ne relèvent pas des études de population et ne sont donc pas identifiées ici.

TABleaU 2 Pourcentage des thèses mentionnant chaque thématique traitée, selon la discipline à laquelle elle se réfère

(\% par rapport au nombre total de thèses, $n=943$ )

\begin{tabular}{|c|c|c|c|c|c|c|c|c|c|c|c|c|}
\hline Thématique & $\begin{array}{l}\frac{0}{60} \\
\frac{0}{0} \\
\frac{0}{U} \\
0 \\
n\end{array}$ & $\begin{array}{l}\frac{0}{\frac{0}{c}} \\
\frac{0}{0} \\
\frac{0}{00} \\
0 \\
\frac{0}{0} \\
0 \\
0\end{array}$ & 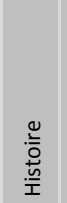 & 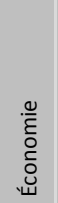 & 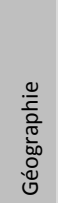 & 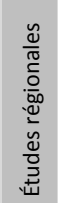 & $\begin{array}{l}\frac{0}{00} \\
\frac{0}{0} \\
\frac{0}{0} \\
\frac{0}{2} \\
\frac{c}{2} \\
\frac{c}{4}\end{array}$ & 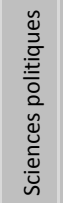 & $\begin{array}{l}\frac{0}{\partial} \\
\frac{0}{0} \\
\frac{0}{3} \\
0 \\
0 \\
\stackrel{0}{0} \\
\stackrel{0}{0} \\
\sim\end{array}$ & : & $\stackrel{0}{\stackrel{一}{*}}$ & 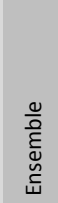 \\
\hline Culture, identité, socialisation et religion & 48,7 & 21,2 & 38,8 & 8,8 & 36,0 & 54,3 & 55,6 & 30,0 & 2,5 & 27,5 & 35,9 & 34,8 \\
\hline Économie, travail, conditions de vie & 22,5 & 36,4 & 20,7 & 75,2 & 36,0 & 20,0 & 9,5 & 24,0 & 7,5 & 12,5 & 20,4 & 29,3 \\
\hline Dynamique des populations & 4,1 & 15,8 & 20,7 & 20,4 & 8,0 & 8,6 & 7,9 & 4,0 & 15,0 & 0,0 & 24,1 & 11,5 \\
\hline Fécondité, famille, sexualité, nuptialité & 26,6 & 35,3 & 17,2 & 18,6 & 7,0 & 11,4 & 23,8 & 8,0 & 7,5 & 32,5 & 20,4 & 22,0 \\
\hline Genre et inégalités hommes femme & 11,2 & 7,6 & 4,3 & 6,2 & 7,0 & 5,7 & 4,8 & 0,0 & 2,5 & 5,0 & 2,9 & 6,9 \\
\hline Méthodologies & 3,4 & 6,5 & 3,4 & 12,4 & 1,0 & 1,4 & 4,8 & 2,0 & 7,5 & 0,0 & 19,4 & 6,4 \\
\hline Migrations & 61,4 & 16,3 & 57,8 & 41,6 & 62,0 & 68,6 & 65,1 & 90,0 & 5,0 & 62,5 & 39,8 & 51,6 \\
\hline Politiques, communication, droit et législation & 24,3 & 22,8 & 39,7 & 24,8 & 20,0 & 37,1 & 27,0 & 72,0 & 2,5 & 65,0 & 17,5 & 29,2 \\
\hline Scolarisation et enseignement & 5,2 & 6,5 & 2,6 & 10,6 & 3,0 & 8,6 & 4,8 & 6,0 & 0,0 & 2,5 & 5,8 & 5,6 \\
\hline Territoire, logement et environnement & 16,1 & 23,4 & 30,2 & 12,4 & 67,0 & 24,3 & 14,3 & 8,0 & 12,5 & 10,0 & 10,7 & 21,1 \\
\hline Mortalité, santé, épidémiologie & 9,4 & 25,5 & 8,6 & 12,3 & 14,0 & 5,7 & 14,3 & 8,0 & 97,5 & 12,5 & 34,0 & 17,6 \\
\hline
\end{tabular}

En vert : valeur significativement supérieure à la moyenne; en rouge : valeur significativement inférieure à la moyenne, par rapport au \% pour l'ensemble des thèses (chi-deux, $p<5 \%$ ).

Note : une thèse peut traiter de plusieurs thématiques.

La démographie constitue une exception en raison d'une absence de thématique dominante. Les thèses réalisées en lien avec cette discipline portent majoritairement sur la fécondité, la famille, la sexualité, la nup- 
tialité d'une part (35\%), les enjeux économiques, de travail et de conditions de vie d'autre part (36\%). La mortalité et la santé sont également très présentes ( $26 \%$ ), bien que dans une moindre mesure, de même que les questions relatives au territoire, au logement, à l'environnement (23\%), à la politique, à la communication, au droit et à la législation ( $23 \%$ ou encore à la culture, l'identité, la socialisation et la religion (21\%). Les autres thématiques sont plus marginales. C'est le cas notamment de la migration (16\%) et la dynamique des populations (16\%). Enfin, moins fréquentes sont les thèses identifiées comme traitant des relations de genre $(8 \%)$, de méthodologie $(7 \%)$, de scolarisation et d'enseignement (7\%).

Ainsi, ces résultats confirment la place privilégiée des thèses sur la fécondité, la nuptialité, la sexualité et la famille, tant par les études en sociologie qu'en démographie. Ils affirment la place spécifique de la démographie parmi les autres sciences de la population.

\section{Ce que nous apprennent les thèses de doctorat sur les études de population}

Les analyses développées ci-dessus offrent un panorama assez large du contenu des thèses de doctorat menées autour de questions de population soutenues en France de 2000 à 2012. Pour constituer notre corpus, nous avons privilégié une étude des termes utilisés dans les titres, des thématiques et des disciplines et cherché à positionner la démographie dans l'ensemble plus vaste des études de population. La procédure de sélection des thèses de doctorat repose sur des choix susceptibles d'être discutés. Il a fallu s'entendre sur ce qu'on entend par «étude dans le domaine de la population». D'une part, la méthode est tributaire du système français de référencement des thèses dans le Sudoc et de la structuration des disciplines au sein de l'université comme nous le verrons plus loin. D'autre part, la variable «discipline» extraite du Sudoc permet d'identifier la discipline telle qu'elle figure sur la page de titre (ou son équivalent) de la thèse. Si cette indication représente le plus souvent la discipline principale dans laquelle est soutenue la thèse, elle peut être plus ou moins précise, notamment lorsqu'elle reprend l'intitulé de l'école doctorale et que celui-ci fait référence à une approche pluridisciplinaire ou une autre discipline plus dominante au sein de l'école (sociologie vs démographie, par exemple). Par ailleurs, on sait que les études de population constituent un champ de recherche éclectique (Maffioli, 
2006a ; 2006b). Les enquêtes menées auprès des membres de l'UIESP montrent que les scientifiques en études de population ont une formation pluridisciplinaire (Loriaux, Vichnevskaia, 2006). Dans l'enquête de 2009, seulement 53 \% des répondants étaient diplômés de démographie mais $71 \%$ se reconnaissaient comme démographes ou population scientists (van Dalen, Henkens, 2012). N'ayant pas de critère objectif précis, la méthode utilisée ici se base sur l'occurrence dans le titre, dans le résumé ou dans les mots-clés indexés d'expressions relevant spécifiquement du champ des études de la population, du moins identifiées comme telles dans le POPIN, le thesaurus qui fait référence dans ce domaine. L'approche que nous avons adoptée a l'avantage de ne pas écarter a priori des thèses sur des critères disciplinaires trop restrictifs, mais elle a probablement dans le même temps élargi le champ disciplinaire. À l'issue de notre sélection, les thèses que nous avons identifiées comme relevant des études de population font surtout référence à la sociologie et en second lieu à la démographie, avec de multiples liens établis avec des disciplines relevant des sciences sociales, des sciences humaines, mais aussi des sciences exactes.

Cette spécificité de la démographie comme une discipline fortement représentée, mais non dominante doit tenir compte du contexte institutionnel français. Bien que la démographie soit récente dans l'histoire des sciences (Vilquin, 2006), la France est en effet caractérisée par une institutionnalisation relativement forte de cette discipline depuis le milieu du 20ème siècle, notamment avec la création en 1945 de l'Institut national d'études démographiques (INED). Parallèlement, des filières et instituts délivrant des diplômes de démographie y compris au niveau doctoral ont été créés. C'est le cas de l'Institut d'études démographiques de l'université de Montesquieu-Bordeaux IV (IEDUB) créé en 1948, de l'Institut de démographie de Paris à l'université de Paris 1 Panthéon Sorbonne (IDUP) fondé en 1957 et de l'Institut de démographie de l'université de Strasbourg (IDUS) créé en 1960. D’autres formations en démographie sont proposées au sein de filières multidisciplinaires des sciences sociales comme à l'université de Paris Ouest Nanterre ou à l'université Paris Descartes, mais dans ce cadre, la démographie est moins mise en avant. De plus, en France, la démographie est regroupée avec la sociologie dans la structuration des disciplines par section qui est établie par le Conseil national universitaire (CNU). Cela peut contribuer à atténuer la visibilité des thèses en démographie.

Dans le domaine des études de population, le choix des sujets de thèse résulte probablement d'un consensus entre l'intérêt de l'étudiant, celui du directeur ou de la directrice de thèse et les ressources potentielles 
liées à la problématique, à la fois en termes de financement, d'accueil au sein d'une structure et de débouchés. Outre l'université, les instituts de recherche français (INED, IRD, CNRS, ...) participent à la formation des doctorants en proposant des laboratoires d'accueil. De fait, ces instituts contribuent largement à structurer les sujets des thèses de doctorat sur la base des projets de recherche. Or, leurs activités sont de plus en plus conditionnées à des sources de financement extérieures (telles que l'Agence nationale la recherche, les programmes de fonds Européens, les organismes multilatéraux, les institutions privées, etc.). A fortiori, les bailleurs de fonds publics ou privés participent aussi et de manière directe à l'orientation de la recherche par des appels à candidatures pour des thèses ciblées sur certains sujets de société (VIH/sida, retraites, politiques familiales, politiques migratoires, ...).

L'un des résultats saillants de nos analyses réside dans la large place occupée par les études sur la migration. La migration est étudiée majoritairement en lien avec deux types de problématiques : les unes relatives à l'identité, la culture et la religion, les autres portants sur l'environnement, le logement et le territoire. L'angle d'approche semble avoir changé par rapport aux décennies 1960, 1970 et 1980. En effet, tel que le souligne Sayad (1984), la majorité des recherches sur la migration, publiées en France en sciences sociales à cette période, mettait en avant la dimension économique des comportements, et plus particulièrement le lien entre immigration et travail. Comme les approches, les thématiques de recherche évoluent probablement au fil du temps. Les thèses soutenues mettent en lumière les thématiques de recherche les plus récentes, au sens où elles sont portées par les chercheurs et universitaires de demain. La large place donnée à la migration dans les études de population peut-elle être vue comme une évolution des intérêts pour la thématique des migrations et des populations (issues) de migrants?

Cette focalisation sur les questions de migration semble rejoindre en partie les préoccupations des membres de l'UIESP enquêtés en 2009 selon lesquels les flux migratoires constituent la seconde thématique prioritaire, après le vieillissement, pour les années à venir en matière de question de population. Cependant, en Europe, le vieillissement des populations est particulièrement mis en avant comme un des enjeux majeurs sur ce continent (van Dalen, Henkens, 2012). Une enquête menée au début des années 2000 sur les centres d'études de la population dans le monde montrait également d'importantes différences entre les continents et les pays. En France, les instituts de démographie mentionnaient des recherches plus orientées sur les dynamiques de population, la ré- 
Myriam de Loenzien, Géraldine Duthé, Lama Kabbanji, Armelle Andro,

Dominique Diguet, Catherine Sluze, Delphine Arnoux

partition sur le territoire, l'organisation sociale, l'économie et les politiques (Gendreau, Huix-Adamets, 2003).

La proportion élevée de thèses sur la migration doit cependant être interprétée en tenant compte de la forte représentation des études de sociologie au sein de cette thématique. En effet, non seulement les migrations sont relativement peu présentes dans les travaux de thèses clairement identifiés comme relevant de la démographie, mais la démographie est également faiblement sollicitée dans les thèses portant sur la migration. Inversement, les études sur la fécondité, la famille, la sexualité ou encore la nuptialité sont caractérisées par un équilibre entre les deux disciplines les plus présentes, la sociologie et la démographie, et un intérêt marqué des démographes. Une étude menée sur les articles parus au début des années 1990 dans Population, la revue de l'INED, montrait une attention particulière pour le thème de la famille (van de Walle, 1995). Cet intérêt n'est de fait pas récent, puisque la fécondité constituait déjà une forte préoccupation des démographes dans la France de l'entre-deux-guerres (Héran, 2013 ; Rosental, 2003). Nous sommes là dans un des domaines privilégiés par les démographes, chercheurs et doctorants, surtout en France.

\section{Conclusion}

Les thèses en étude de population dans leur ensemble sont caractérisées par le recours principalement à des disciplines de sciences sociales, voire des sciences humaines et plus rarement des approches biomédicales ou de sciences exactes. Les deux disciplines principales auxquelles ces études font appel sont en premier lieu la sociologie et dans une moindre mesure la démographie, tant du point de vue du nombre de thèses concernées que de la centralité de la discipline au sein des thèses d'approche pluridisciplinaire.

En termes de thématiques, notre corpus affirme la place prépondérante de la migration au sein des études de population, mais dans un contexte de lien avec la sociologie plutôt que la démographie. Il confirme en revanche l'intérêt des thèses de démographie pour la fécondité, la famille, la nuptialité, la sexualité. Les thèses portant sur la mortalité, la santé, la dynamique de population adoptent quant à elles des approches plus quantitatives et mettent davantage en œuvre des modélisations, avec un accent sur des aspects méthodologiques. Ces tendances se dégagent dans un contexte où de nombreuses études proposent des positionne- 
ments intermédiaires, rendant impossible de dresser des lignes tranchées et mettant en avant le caractère relatif de toute catégorisation.

Sociologie et démographie occupent des positions très fortes et relativement équilibrées au sein des études sur la fécondité, la famille, la nuptialité, la sexualité. Cet équilibre entre sociologie et démographie est également présent dans d'autres travaux, où dominent néanmoins d'autres sciences sociales. C'est le cas notamment des thèses traitant de sujets économiques sur le travail, les conditions de vie ou sur l'éducation et l'enseignement. C'est le cas également de travaux comportant une dimension plus clairement géographique sur l'environnement, le logement, et le territoire. En revanche, la sociologie prédomine clairement dans les études de genre ainsi que dans les thèses sur la migration ou encore sur la culture, l'identité, les minorités, et la religion. À l'inverse, la démographie est plus présente dans les études sur la dynamique de population ainsi que sur la mortalité et la santé. La situation des autres thématiques est plus contrastée.

L'analyse approfondie de l'évolution des thématiques de thèses pourra être menée à travers des prolongements possibles de ce travail. L'un d'eux concerne une exploration des thèses plus anciennes, ce qui est en cours dans le cadre de ce projet grâce à l'incorporation des thèses soutenues avant les années 2000. Une telle étude est complexe, car elle nécessite de prendre en compte plusieurs évolutions simultanées. En effet, l'évolution du nombre de thèses doit être mise en parallèle avec les changements de thématiques, le développement des disciplines, la préférence plus ou moins marquée selon les périodes pour une approche plurithématique ou pluridisciplinaire. Un autre volet consiste à approfondir l'analyse des thèses portant sur la migration, exploration également en cours. L'analyse des champs disciplinaires et des thèmes de recherche pourrait en outre être complétée par une étude plus institutionnelle des thèses soutenues dans le domaine des études de population en France. Celle-ci constituerait un approfondissement d'une première description déjà réalisée (Andro et al., 2013 ; Arnoux, 2013). Elle aiderait à préciser l'influence du cadre de réalisation de la thèse via le développement des formations dispensées au sein des écoles doctorales, le rayonnement des travaux scientifiques de certains directeur.trice.s de thèse, l'appui accordé à certaines thématiques. Une autre voie d'approfondissement possible consiste à étudier les zones géographiques étudiées dans ces thèses, disponible dans la base. Enfin, des analyses comparées avec la production de thèses d'études des populations dans d'autres pays pourraient à terme, considérablement enrichir notre connaissance des études de population au niveau international. 
Myriam de Loenzien, Géraldine Duthé, Lama Kabbanji, Armelle Andro,

Dominique Diguet, Catherine Sluze, Delphine Arnoux

\section{Références}

Andro A., Arnoux D., Diguet D. C., Duthé G., Kabbanji L., Lesclingand M., Loenzien M. DE, SLUSE C. E. (2013), «Becoming a Population Scientist in the 21st Century: Exploratory Analysis of the French Case», Communication orale présentée à la conférence internationale de population, UIESP/IUSSP, Busan.

ARNoux D. (2013), Les thèses en sciences de la population en France : caractéristiques, place de la démographie et évolution depuis 2000, Université Paris Panthéon Sorbonne, Rapport de stage, IDUP, Paris, $64 \mathrm{p}$.

BARIBEAU C. (2007), «Panorama du développement de la recherche qualitative: analyse critique des thèses produites en sciences humaines et sociales depuis dix ans (1996-2005), en langue française dans les universités francophones au Québec», Recherches Qualitatives, 27 (2), pp. 28-37.

BENABOU-LuCIDO L. (2011), "Histoire du développement de la recherche universitaire française sur les migrations internationales (1815-1999)», Revue Européenne des Migrations Internationales, 27 (3), pp. 7-30, http://dx.doi.org/10.4000/remi.5609.

CICRED (Comité International de Coopération dans les Recherches nationales en Démographie) (1993), Thesaurus de POPIN : thesaurus multilingue de population, 3ème édition, POPIN/CICRED/FNUAP, Paris, $266 \mathrm{p}$.

Gendreau F., Huix-Adamets S. (2003), Panorama institutionnel et scientifique de la recherche démographique dans le monde, Comité International de Coopération dans les Recherches nationales en Démographie (CICRED), Paris, 99 p.

Guérin-PACE F., SAINT-JuLien T. (2012), «Une analyse lexicale des titres et mots-clés de 1972 à 2010», L'espace Géographique, 1 (41), Belin édition, pp. 4-30.

HÉRAN F. (2013), "La démographie et son vocabulaire au fil des siècles : une exploration numérique», Population \& Sociétés, (505), 4 p.

LoRiaux M., Vichnevskaia T. (2006), «La démographie vue par ceux qui la font. Simples considérations sur la démographie et les démographes à partir d'une enquête électronique internationale réalisée en 2000», G. CASELLI, J. VALLIN, G. WUNSCH (eds), Démographie. Analyse et synthèse VIII. Observation, Méthodes auxiliaires, Enseignement et Recherche, Chapitre 143, pp. 693-728.

MAFFIOLI D. (2006a), «Enseignement et recherche en démographie», G. Caselli, J. Vallin, G. Wunsch (eds), Démographie. Analyse et synthèse VIII. Observation, Méthodes auxiliaires, Enseignement et Recherche, Chapitre 140, pp. 603-634.

MAfFIOL D. (2006b), «Centres de recherche et d'enseignement démographiques : naissance, développement, tendances actuelles», G. Caselli, J. Vallin, G. Wunsch (eds), Démographie. Analyse et synthèse VIII. Observation, Méthodes auxiliaires, Enseignement et Recherche, Chapitre 142, pp. 659-692. 
Marchand P., Ratinaud P. (2012), "L'analyse de similitude appliquée aux corpus textuels: les primaires socialistes pour l'élection présidentielle française», Actes des 11èmes Journées internationales d'Analyse statistique des Données Textuelles. JADT, Liège, pp. 687-699.

Rosental P.-A. (2003), L'Intelligence démographique : sciences et politiques des populations en France (1930-1960), Paris, Odile Jacob, 368 p.

SAYAD A. (1984), "Tendances et courants des publications en sciences sociales sur I'immigration en France depuis 1960», Current Sociology, (32) 3, pp. 219-304, http://dx.doi.org/10.1177/001139284032003004.

VAN Dalen H., Henkens K. (2012), "What is on a Demographer's Mind? A Worldwide Survey», Demographic Research, (26), pp.363-408, http://dx.doi.org/10.4054/ DemRes.2012.26.16.

VAN DE WALLE E. (1995), «Les particularités de Population dans les thèmes traités et les méthodes utilisées», Population, 6, pp. 1 445-1 456, http://dx.doi.org/10.2307/ 1534461.

VILQUIN E. (2006), "Les origines de la science démographique», G. Caselli, J. Vallin, G. Wunsch (eds), Démographie. Analyse et synthèse VIII. Observation, Méthodes auxiliaires, Enseignement et Recherche, Chapitre 138, pp. 507-526.

\section{Remerciements}

Les auteures ont mené cette recherche dans le cadre du CNF de l'UIESP. Elles remercient l'UIESP, l'INED et l'IRD pour leur aide et leur soutien, ainsi que Kadir Elmi Cheik pour son appui technique. Elles remercient également les relecteurs de la Revue Quetelet pour leurs remarques et suggestions qui ont permis des améliorations significatives de cet article. 


\section{Annexe 1.}

Mots-clés RAMEAU utilisés pour la recherche dans le Sudoc

Population (restriction à la population humaine et ses subdivisions, par exemple : densité, aspect économique etc.), démographes, démographie et toutes les expressions contenant «démographie ou démographique ou démographiques», Malthus, Malthusianisme, paléodémographie, surpopulation, vieillissement de la population, avortement, contraception, régulation des naissances, santé de la reproduction, fécondité humaine, mariage, divorce, décès causes, mortalité, morbidité, espérance de vie, transition épidémiologique, eugénisme, émigration, immigration, immigrées, migrations intérieures, diasporas, étrangers, enfants d'immigrés, enfants des baby-boomers, famille monoparentale, famille recomposée, familles immigrées, recensement, statistiques de l'état civil.

\section{Annexe 2.}

Liste des disciplines explicitement mentionnées sur la page de couverture des thèses et nombre de thèses concernées

\begin{tabular}{|l|c|}
\hline Discipline & $\begin{array}{c}\text { Nombre de thèses } \\
\text { mentionnant la discipline }\end{array}$ \\
\hline Sociologie & 267 \\
\hline Démographie & 184 \\
\hline Histoire & 116 \\
\hline Économie & 113 \\
\hline Géographie & 100 \\
\hline Études régionales & 70 \\
\hline Anthropologie & 63 \\
\hline Sciences Politiques & 50 \\
\hline Droit & 40 \\
\hline Santé publique & 40 \\
\hline Lettres & 25 \\
\hline Biologie & 21 \\
\hline Psychologie & 15 \\
\hline Gestion & 12 \\
\hline Médecine & 12 \\
\hline Mathématiques & 8 \\
\hline Philosophie & 4 \\
\hline Statistiques & 4 \\
\hline Archéologie & 2 \\
\hline Écologie & 2 \\
\hline Art & 152 \\
\hline Informatique & 1 \\
\hline Linguistique & 1 \\
\hline Pharmacie & 2 \\
\hline Total de mentions (sur 943 thèses) & 13 \\
\hline & 2 \\
\hline
\end{tabular}




\section{Annexe 3.}

Résultats de l'analyse en composantes principales pour les 5 premières composantes : valeurs propres initiales et matrice

\begin{tabular}{|c|c|c|c|c|c|}
\hline & \multicolumn{5}{|c|}{ Composante } \\
\hline & 1 & 2 & 3 & 4 & 5 \\
\hline Variance & 1,955 & 1,446 & 1,189 & 1,116 & $5 \quad 1,037$ \\
\hline$\%$ de la variance totale & 17,8 & 13,1 & 10,8 & 10,1 & 9,4 \\
\hline \% cumulé & 17,8 & 30,9 & 41,7 & 51,9 & 61,2 \\
\hline Culture, identité, socialisation et religion & 0,604 & 0,182 & $-0,275$ & 0,203 & $3 \quad 0,363$ \\
\hline Démographie économique, travail, conditions de vie & $-0,001$ & $-0,441$ & 0,671 & 0,052 & $-0,306$ \\
\hline Dynamique des populations & $-0,534$ & $-0,278$ & $-0,085$ & 0,066 & $5-0,011$ \\
\hline Fécondité, famille, sexualité, nuptialité & $-0,212$ & 0,719 & 0,167 & 0,274 & $-0,148$ \\
\hline Genre et inégalités hommes femmes & 0,061 & 0,545 & 0,178 & 0,243 & $3 \quad 0,013$ \\
\hline Méthodologies & $-0,426$ & $-0,161$ & $-0,076$ & $-0,213$ & $3 \quad 0,550$ \\
\hline Migrations & 0,768 & $-0,264$ & $-0,056$ & $-0,108$ & $3 \quad 0,185$ \\
\hline Politiques, communication, droit et législation & 0,339 & 0,104 & $-0,203$ & $-0,578$ & $3-0,534$ \\
\hline Scolarisation et enseignement & 0,108 & 0,029 & 0,660 & $-0,205$ & 0,375 \\
\hline Territoire, logement et environnement & 0,004 & $-0,464$ & $-0,211$ & 0,652 & $-0,159$ \\
\hline Mortalité, santé, épidémiologie & $-0,598$ & 0,070 & $-0,258$ & $-0,273$ & $3 \quad 0,040$ \\
\hline
\end{tabular}

Annexe 4.

Caractérisation des principaux groupes de thèses : classes comprenant au moins 20 thèses (voir Figure 4)

\begin{tabular}{|c|c|l|}
$\begin{array}{c}\text { Rang du } \\
\text { groupe }\end{array}$ & $\begin{array}{c}\text { Nombre } \\
\text { de thèses }\end{array}$ & \multicolumn{1}{|c|}{ Disciplines dominantes } \\
\hline 1 & 78 & Sociologie \\
\hline 2 & 46 & Sociologie, pluridisciplinarité \\
\hline 3 & 44 & Sciences politiques \\
\hline 4 & 42 & Pas de discipline dominante \\
\hline 5 & 32 & Démographie et disciplines autres \\
\hline 6 & 29 & Economie et pluridisciplinarité \\
\hline 7 & 27 & Démographie, sociologie et disciplines autres \\
\hline 8 & 23 & Démographie \\
\hline 10 & 22 & Géographie \\
\hline 11 & 21 & Sciences politiques, économie, pluridisciplinarité très marquée \\
\hline & 20 & Sociologie, géographie, démographie, pluridisciplinarité \\
\hline
\end{tabular}

